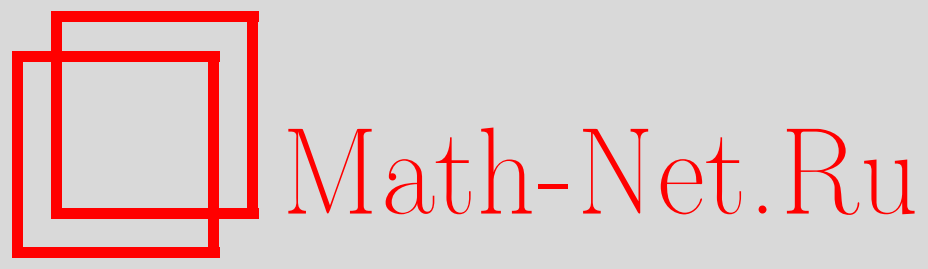

А. Ю. Перепечко, Гибкость аффинных конусов над поверхностями дель Пеццо степени 4 и 5 , Функи. анализ и его прил., 2013, том 47, выпуск 4, 45-52

DOI: https://doi.org/10.4213/faa3127

Использование Общероссийского математического портала MathNet.Ru подразумевает, что вы прочитали и согласны с пользовательским соглашением

http://www . mathnet.ru/rus/agreement

Параметры загрузки:

IP : 52.6 .47 .48

26 апреля 2023 г., 15:07:47

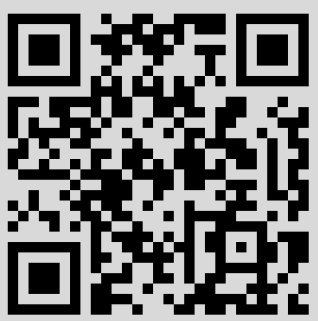


Функционалъный анализ и его приложения

2013, т. 47, вып. 4, с. 45-52

УДК 512.745.4

\title{
Гибкость аффинных конусов над поверхностями дель Пеццо степени 4 и 5*
}

\author{
(C) 2013. А. Ю. ПЕРЕПЕчКО
}

В работе доказана бесконечная транзитивность действия группы специальных автоморфизмов аффинных конусов над поверхностями дель Пеццо степени 4 и 5 .

\section{1. Введение}

Аффинное алгебраическое многообразие $X$, определенное над алгебраически замкнутым полем $\mathbb{K}$ характеристики нуль, называется гибким, если касательное пространство к $X$ в произвольной гладкой точке порождается касательными векторами к орбитам действия однопараметрических унипотентных групп [3]. В этой работе мы установим гибкость аффинных конусов над поверхностями дель Пеццо степени 4 и 5.

Известно, что каждому действию одномерной унипотентной группы $\mathbb{G}_{a}=$ $\mathbb{G}_{a}(\mathbb{K})$ на $X$ соответствует локально нильпотентное дифференцирование $\delta \in$ $\operatorname{LND}(\mathbb{K}[X])$ алгебры регулярных функций на $X$. Все такие действия порождают подгруппу специальных автоморфизмов SAut $X \subset$ Aut $X$.

Говорят, что группа $G$ действует на множестве $X$ бесконечно транзитивно, если для любого $m \in \mathbb{N}$ она действует транзитивно на множестве упорядоченных наборов из $m$ попарно различных точек множества $X$.

Следующая теорема объясняет значение условия гибкости.

Теорема 1 [3, теорема 0.1]. Пусть X - неприводимое аффинное алгебраическое многообразие размерности $\geqslant 2$. Тогда следующие условия эквивалентны:

(1) многообразие $X$ является гибким;

(2) группа SAut $X$ действует транзитивно на множестве гладких точек $X_{\text {reg }}$;

(3) группа SAut $X$ действует бесконечно транзитивно на $X_{\mathrm{reg}}$.

В [4] описаны три класса гибких аффинных многообразий, а именно аффинные конусы над многообразиями флагов, невырожденные торические многообразия размерности $\geqslant 2$ и надстройки над гибкими многообразиями. Заметим, что аффинные конусы над поверхностями дель Пеццо степени $\geqslant 6$ являются торическими, а значит, гибкими многообразиями.

В данной работе мы рассматриваем случаи степени 4 и 5 . Для степени 5 мы доказываем гибкость аффинных конусов, соответствующих поляризациям по любым очень обильным дивизорам, а для степени 4 - по некоторым, в том числе по антиканоническому дивизору. Как показано соответственно в $[5$, теорема

*Работа выполнена при поддержке ФЦП «Научные и научно-педагогические кадры инновационной России» на 2009-2013 годы (соглашение № 8214) и РФФИ (гранты № 12-01-00704 и №12-01-31342мол_а). 
1.1] и в [8, следствие 1.8], аффинные конусы над антиканоническим вложением поверхностей дель Пеццо степени 3 и конусы над плюриантиканоническим вложением для степени $\leqslant 2$ не обладают ни одним $\mathbb{G}_{a}$-действием.

В доказательстве мы используем конструкцию из [7], которая по каждому открытому цилиндрическому подмножеству некоторого специального вида в проективном многообразии $Y$ позволяет построить регулярное действие группы $\mathbb{G}_{a}$ на аффинном конусе над $Y$. В терминах трансверсального покрытия такими подмножествами в теореме 5 получено достаточное условие гибкости аффинного конуса над проективным многообразием, применяемое впоследствии к поверхностям дель Пеццо.

Автор благодарен М. Г. Зайденбергу за постановку задачи и многочисленные обсуждения, И. В. Аржанцеву за ценные замечания и рецензенту за исправление ряда неточностей.

\section{2. Гибкость аффинных конусов}

Пусть $Y$ - проективное многообразие, а $H$ - очень обильный дивизор на $Y$. Поляризация многообразия $Y$ по $H$ задает вложение $Y \hookrightarrow \mathbb{P}^{n}$. Рассмотрим aффинный конус $X=$ AffCone $_{H} Y \subset \mathbb{A}^{n+1}$ с вершиной в начале координат $0 \in \mathbb{A}^{n+1}$, отвечающий этому вложению. На нем имеется естественное действие гомотетиями мультипликативной группы $\mathbb{G}_{m}=\mathbb{G}_{m}(\mathbb{K})$ поля $\mathbb{K}$. Оно задает градуировку алгебры $\mathbb{K}[X]$. Дифференцирование на $\mathbb{K}[X]$ называется однородным, если оно переводит однородные элементы в однородные. Подмножество однородных локально нильпотентных дифференцирований обозначают через $\operatorname{HLND}(\mathbb{K}[X])$.

Определение 2 [7, определения 3.1.4, 3.1.7]. Будем говорить, что открытое подмножество $U$ многообразия $Y$ является иилиндром, если $U \cong Z \times \mathbb{A}^{1}$, где $Z$ - гладкое многообразие и $\operatorname{Pic} Z=0$. Пусть теперь дан дивизор $H \subset Y$. Цилиндр $U$ будем называть $H$-полярным, если $U=Y \backslash \operatorname{supp} D$ для некоторого эффективного дивизора $D \in|d H|$, где $d>0$.

Определение 3. Подмножество $W \subset Y$ будем называть инвариантным относительно цилиндра $U=Z \times \mathbb{A}^{1}$, если $W \cap U=\pi_{1}^{-1}\left(\pi_{1}(W)\right)$, где $\pi_{1}: U \rightarrow Z-$ проекция на первую компоненту прямого произведения. Иначе говоря, каждый $\mathbb{A}^{1}$-слой цилиндра либо содержится в $W$, либо не пересекается с $W$.

Определение 4. Будем говорить, что многообразие $Y$ покрывается трансверсально цилиндрами $U_{i}, i=1, \ldots, s$, если $\bigcup U_{i}=Y$ и не существует собственного подмножества $W \subset Y$, инвариантного относительно всех $U_{i}$.

Ясно, что каждый цилиндр $U_{i}$ является гладким. Значит, особое многообразие $Y$ не допускает трансверсального покрытия цилиндрами. Ясно также, что $\operatorname{dim} Y \geqslant 1$. В следующей теореме мы даем достаточное условие гибкости аффинного конуса над проективным вложением $Y \hookrightarrow \mathbb{P}^{n}$, отвечающим поляризации $H$.

Теорема 5. Предположим, что для некоторого очень обильного дивизора Н на гладком проективном многообразии $Y$ существует трансверсальное покрытие $H$-полярными иилиндрами. Тогда афбфинный конус $X=\operatorname{AfCone}_{H} Y$ является гибким. 
Доказательство. Утверждение очевидно для $X=\mathbb{A}^{n+1}$. Поэтому мы можем считать, что вершина конуса является его единственной особой точкой.

Согласно [7, теорема 3.1.9], каждому цилиндру покрытия $Y$ соответствует однородное $\mathbb{G}_{a}$-действие на $X$. Из явного построения в [7, предложение 3.1.5] видно, что орбиты этого действия при проекции $\pi: X^{\times}=X \backslash\{0\} \rightarrow Y$ переходят в слои цилиндра на $Y$, а множество неподвижных точек на $X$ - это прообраз дополнения к цилиндру.

Пусть $G \subset$ SAut $X$ - подгруппа, порожденная соответствующими $\mathbb{G}_{a}$-действиями. Рассмотрим орбиту $G x$ некоторой точки $x \in X^{\times}$. Ее образ $\pi(G x) \subset Y$ будет подмножеством, инвариантным относительно всех цилиндров покрытия. Из условия транверсальности получаем $\pi(G x)=Y$. Поскольку группа $G$ порождена однородными действиями, естественное $\mathbb{G}_{m}$-действие на $X$ гомотетиями нормализует действие $G: X$ и переводит $G$-орбиты в $G$-орбиты. А значит, $X^{\times}$является объединением $G$-орбит, проекция каждой из которых совпадает со всем $Y$. Следовательно, $X^{\times}=\bigcup_{\lambda \in \mathbb{G}_{m}} \lambda G x$, где все $G$-орбиты замкнуты в $X^{\times}$.

Покажем, что на самом деле имеется одна открытая $G$-орбита $G x=X^{\times}$. Предположим противное. В таком случае $\operatorname{dim} G x=\operatorname{dim} Y$ и стабилизатор $S \subset$ $\mathbb{G}_{m}$ орбиты $G x$ конечен. Более того, поскольку действие $S: G x$ свободно, для любой точки $x^{\prime} \in G x$ пересечение $G x \cap \mathbb{G}_{m} x^{\prime}$ есть $S$-орбита, состоящая из $|S|$ различных точек. Раздутие конуса $X$ в вершине является тотальным пространством линейного расслоения $[-H]$ на $Y$. Оно естественным образом пополняется до $\mathbb{P}^{1}$-расслоения $\widehat{X} \rightarrow Y$. Для общей точки $x^{\prime} \in G x$ пересечение $\overline{G x} \cap \overline{\mathbb{G}_{m} x^{\prime}}$, где $\bar{Z}$ обозначает замыкание $Z \subset X^{\times}$в $\widehat{X}$, совпадает с орбитой $S x$. Значит, индекс пересечения $\overline{G x} \cdot \overline{\mathbb{G}_{m} x^{\prime}}$ равен $|S|$. Поскольку индекс пересечения постоянен, для любой точки $x^{\prime} \in G x$ имеем $\overline{G x} \cap \overline{\mathbb{G}_{m} x^{\prime}}=S x^{\prime} \subset X^{\times}$. Таким образом, квазиаффинное многообразие $X^{\times}$содержит проективное $\overline{G x}$. Противоречие. Значит, группа $G$ действует на $X^{\times}$транзитивно.

\section{§3. Поверхность дель Пеццо степени 5}

Пусть $Y$ - поверхность дель Пеццо степени 5. Она получается раздутием четырех точек $P_{1}, \ldots, P_{4}$ на проективной плоскости $\mathbb{P}^{2}$, никакие три из которых не лежат на одной прямой [1, теорема IV.2.5]. Поскольку автоморфизмы проективной плоскости действуют транзитивно на таких четверках точек, поверхность единственна с точностью до изоморфизма.

Теорема 6. Пусть $H$ - произвольный оченъ обилъный дивизор на поверхности дель Пецио $Y$ степени 5. Тогда афбинный конус AffCone $_{H} Y$, отвечающий Н, является гибким.

Доказательство проводится в несколько этапов, см. пп. 3.1 и 3.2. Через $E_{i}$ будем обозначать исключительные дивизоры - прообразы точек раздутия $P_{i}$. Пусть $e_{0}$ - класс дивизоров, эквивалентных прямой, не проходящей через точки раздутия, а $e_{i}(i=1, \ldots, 4)$ - класс дивизоров, эквивалентных $E_{i}$. Эти классы порождают группу Пикара $\operatorname{Pic~} Y=\left\langle e_{0}, \ldots, e_{4}\right\rangle_{\mathbb{Z}} \cong \mathbb{Z}^{5}$. Индекс пересечения определяет симметричную билинейную форму на группе Пикара, причем набор $\left\{e_{0}, \ldots, e_{4}\right\}$ является ортогональным базисом с $e_{0}^{2}=1, e_{i}^{2}=-1$. Классы исключительных дивизоров (или, иначе говоря, (-1)-кривых) исчерпываются классами $e_{i}$ и $e_{0}-e_{i}-e_{j}$ для различных $i, j \neq 0$. 
По критерию обильности Клеймана [9, теорема 1.4.9] замыкание конуса обильных дивизоров Ample $Y$ является двойственным к конусу эффективных дивизоров $\overline{\mathrm{NE}}(Y)$. В случае поверхностей дель Пеццо степени $<8$ конус $\overline{\mathrm{NE}}(Y)$ порожден исключительными дивизорами, см. [6, теорема 8.2.19]. Следовательно, конус обильных дивизоров задается неравенствами

$$
\begin{gathered}
x_{0}>0, \quad x_{i}<0, \quad i=1, \ldots, 4, \\
x_{0}+x_{i}+x_{j}>0, \quad 0 \neq i \neq j \neq 0,
\end{gathered}
$$

где $\left(x_{0}, \ldots, x_{4}\right) \in \mathrm{Pic} Y$. У него всего десять экстремальных лучей - это

$$
e_{0}, e_{0}-e_{j}, 2 e_{0}-\sum_{i \neq 0} e_{i}, 2 e_{0}-\sum_{i \neq 0, j} e_{i} \quad \text { при } j=1, \ldots, 4 .
$$

Для пяти из них в соответствующей ортогональной гиперграни конуса эффективных дивизоров лежит четверка непересекающихся $(-1)$-кривых. Она задает стягивание $Y \rightarrow \mathbb{P}^{2}$, соответствующее выбранному экстремальному лучу.

Каждый луч из оставшихся пяти определяет пучок квадрик на $Y$. А именно, в ортогональном дополнении к лучу лежат три пары пересекающихся $(-1)$-кривых, образующих вырожденные слои пучка квадрик. При этом класс слоя пучка лежит на выбранном луче.

3.1. Цилиндры. Зафиксируем стягивание $\varphi: Y \rightarrow \mathbb{P}^{2}$ четырех непересекающихся $(-1)$-кривых $E_{1}, \ldots, E_{4}$ в точки $P_{1}, \ldots, P_{4}$ и используем обозначения, введенные выше. Пусть $l_{i j} \subset \mathbb{P}^{2}$ - прямая, проходящая через точки $P_{i}$ и $P_{j}$. Рассмотрим открытое подмножество $U_{1}=\varphi^{-1}\left(\mathbb{P}^{2} \backslash\left(l_{12} \cup l_{34}\right)\right) \subset Y$. Это цилиндр, определяемый пучком прямых, проходящих через базисную точку $\operatorname{Bs}\left(U_{1}\right)=l_{12} \cap l_{34}$, причем $U_{1} \cong \mathbb{A}_{*}^{1} \times \mathbb{A}^{1}$, где $\mathbb{A}_{*}^{1}=\mathbb{A}^{1} \backslash\{0\}$. Аналогично положим $U_{2}=\varphi^{-1}\left(\mathbb{P}^{2} \backslash\left(l_{13} \cup l_{24}\right)\right)$ и $U_{3}=\varphi^{-1}\left(\mathbb{P}^{2} \backslash\left(l_{14} \cup l_{23}\right)\right)$, как на рис. 1 . Рассмотрим далее стягивания других четверок непересекающихся (-1)-кривых на $Y$. Всего таких наборов пять, см. рис. 2. Каждому стягиванию соответствует три цилиндра, получаемых аналогично. Заметим, что таким цилиндрам взаимно однозначно соответствуют точки пересечения $(-1)$-кривых и группа автоморфизмов Aut $Y \cong S_{5}$ действует на их множестве транзитивно.
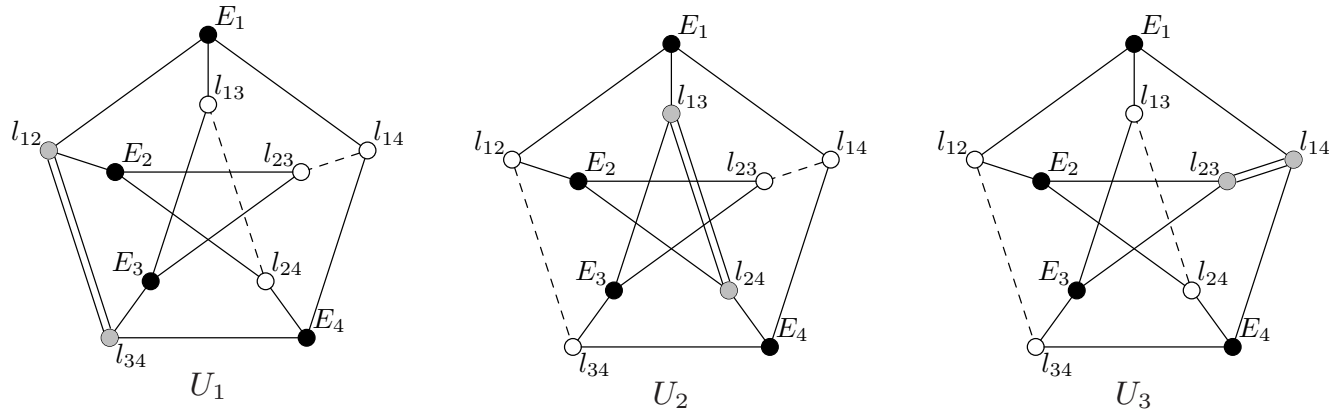

Рис. 1. Расположение цилиндров на графе инцидентности $(-1)$-кривых на поверхности дель Пеццо степени 5. Серым и черным точкам соответствуют (-1)-кривые, лежащие в дополнении к цилиндру. Ребра, нарисованные пунктиром, отвечают пересечениям (-1)-кривых, принадлежащим цилиндру. Двойному ребру соответствует базисная точка соответствующего цилиндра. 


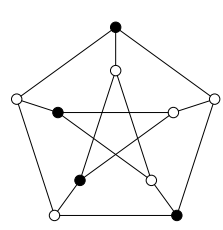

$U_{1}, U_{2}, U_{3}$

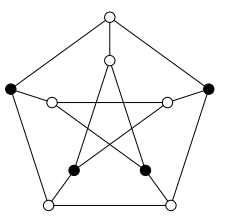

$U_{4}, U_{5}, U_{6}$

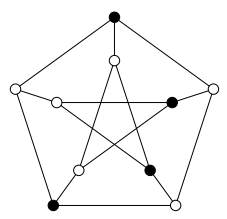

$U_{7}, U_{8}, U_{9}$

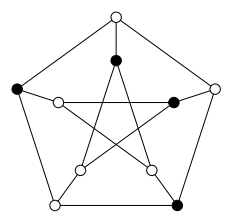

$U_{10}, U_{11}, U_{12}$

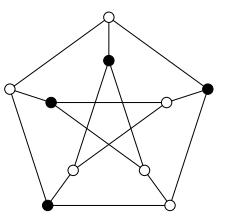

$U_{13}, U_{14}, U_{15}$

Рис. 2. Черным точкам отвечают стягиваемые четверки (-1)-кривых.

По каждому стягиванию строится три цилиндра, как на рис. 1.

Итак, мы определили цилиндры $U_{1}, \ldots, U_{15}$ такого вида, как показано на рис. 1 и 2. Несложно проверить, что каждое пересечение $(-1)$-кривых лежит в каком-то цилиндре; поэтому $\bigcup U_{i}=Y$. Мы утверждаем, что не найдется собственного подмножества $W \subset Y$, инвариантного относительно всех 15 цилиндров. Предположим противное: пусть такое $W$ нашлось. Возьмем произвольную точку в $W$, она содержится в слое $S$ некоторого цилиндра. Тогда $W$ содержит $S$. Без ограничения общности можно считать, что $S-$ слой цилиндра $U_{1}$. Тогда прямая $l=\overline{\varphi(S)} \subset \mathbb{P}^{2}$ проходит через базисную точку $\operatorname{Bs}\left(U_{1}\right)$. Поскольку точки $\operatorname{Bs}\left(U_{1}\right), \operatorname{Bs}\left(U_{2}\right), \operatorname{Bs}\left(U_{3}\right)$ не лежат на одной прямой, одна из них не лежит на $l$. Пусть $\operatorname{Bs}\left(U_{2}\right) \notin l$. Тогда слой $S$ пересекается почти со всеми слоями цилиндра $U_{2}$ и множество $W$ содержит почти все слои этого цилиндра, т. е. плотно в $Y$. Дополнение $Y \backslash W$ также инвариантно относительно всех цилиндров, и по тем же причинам оно плотно в $Y$, что невозможно.

3.2. Условие полярности. Здесь мы установим, что для любого обильного дивизора $H$ на $Y$ все 15 цилиндров $U_{i}$ являются $H$-полярными. Рассмотрим множество эффективных дивизоров $\left\{\sum_{i s=1}^{4} \alpha_{i} E_{i}+\beta_{1} l_{12}+\beta_{3} l_{34} \mid \alpha_{i}, \beta_{i}>0\right\}$, дополнение к носителю которых совпадает с $U_{1}$. В группе Пикара ему соответствует открытый конус $C$, экстремальные лучи которого суть $e_{1}, e_{2}, e_{3}, e_{4}$, $e_{0}-e_{1}-e_{2}, e_{0}-e_{3}-e_{4}$. Легко проверить, что примитивные векторы обильного конуса (3) выражаются как линейные комбинации с неотрицательными рациональными коэффициентами примитивных векторов конуса $C$. Следовательно, цилиндр $U_{1}$ является $H$-полярным для любого обильного дивизора $H$. Действуя автоморфизмами из Aut $Y$, мы можем перевести $U_{1}$ в любой цилиндр. Поэтому все $U_{i}$ будут $H$-полярными для любого обильного дивизора $H$. Применяя теорему 5, приходим к требуемому утверждению. Теорема 6 доказана.

\section{4. Поверхности дель Пеццо степени 4}

Каждая поверхность дель Пеццо степени 4 изоморфна раздутию проективной плоскости $\mathbb{P}^{2}$ в пяти точках, никакие три из которых не лежат на одной прямой. Такие поверхности образуют двупараметрическое семейство.

Через $E_{i}$ будем обозначать $(-1)$-кривые - прообразы точек раздутия $P_{i}$. Как и прежде, пусть $e_{0}$ - класс дивизоров, эквивалентных прямой, не проходящей через точки раздутия, а $e_{i}(i=1, \ldots, 5)$ - класс дивизоров, эквивалентных $E_{i}$. Набор $\left\{e_{0}, \ldots, e_{5}\right\}$ является ортогональным базисом в группе Пикара $\operatorname{Pic~} Y \cong$ $\mathbb{Z}^{6}$, причем $e_{0}^{2}=1, e_{i}^{2}=-1$. Классы исключительных дивизоров исчерпываются классами $e_{i}, e_{0}-e_{i}-e_{j}, 2 e_{0}-\sum_{k \neq 0} e_{k}$ для различных $i, j \neq 0$. Конус обильных 
дивизоров задается неравенствами

$$
\begin{gathered}
x_{0}>0, \quad x_{i}<0, \quad i=1, \ldots, 5, \\
x_{0}+x_{i}+x_{j}>0, \quad 0 \neq i \neq j \neq 0, \\
2 x_{0}+x_{1}+\cdots+x_{5}>0,
\end{gathered}
$$

где $\left(x_{0}, \ldots, x_{5}\right) \in \operatorname{Pic} Y$. Его экстремальные лучи - это

$$
\begin{array}{r}
e_{0}, e_{0}-e_{j}, 2 e_{0}-\sum_{k \neq 0, i} e_{k}, 2 e_{0}-\sum_{k \neq 0, i, j} e_{k}, 3 e_{0}-\sum_{k \neq 0} e_{k}-e_{i} \\
\text { при различных } 1 \leqslant i, j \leqslant 5 .
\end{array}
$$

Как и в случае поверхности дель Пеццо степени 5 , шестнадцать экстремальных лучей соответствуют стягиваниям $Y \rightarrow \mathbb{P}_{2}$, а десять - пучкам квадрик на $Y$.

4.1. Цилиндры. Для некоторой $(-1)$-кривой $C_{1}$ рассмотрим стягивание $\sigma_{1}: Y \rightarrow \mathbb{P}^{2}$ пяти $(-1)$-кривых $F_{1}, \ldots, F_{5}$, пересекающих $C_{1}$, см. рис. 3. Оно корректно определено, поскольку стягиваемые дивизоры не пересекаются. Образ $\sigma_{1}\left(C_{1}\right)$ - это гладкая квадрика $c$, проходящая через центры раздутия $Q_{1}, \ldots, Q_{5}$. Возьмем произвольную прямую $l \subset \mathbb{P}^{2}$, касающуюся $c$ в точке, отличной от $Q_{1}, \ldots, Q_{5}$. Пучок квадрик в $\mathbb{P}^{2}$, порожденный дивизорами $c$ и $2 l$, определяет цилиндр $U \cong \mathbb{A}_{*}^{1} \times \mathbb{A}^{1} \subset Y$, дополнение к которому есть полный прообраз носителя дивизора $c+2 l$ в $\mathbb{P}^{2}$. Обозначим через $\mathscr{U}_{C_{1}}$ семейство таких цилиндров в $Y$ при всевозможных выборах касательной $l$ к $c$. Заметим, что $Y \backslash \bigcup_{U \in \mathscr{\ell}_{C_{1}}} U$ есть объединение $C_{1}$ и стягиваемых дивизоров $F_{i}(i=1, \ldots, 5)$. $\mathrm{C}$ помощью аналогичной конструкции для $(-1)$-кривых $C_{2}, \ldots, C_{5}$, образующих с $C_{1}$ цикл длины 5 на графе инцидентности, см. рис. 3 , мы получаем в итоге пять семейств цилиндров $\mathscr{U}_{C_{1}}, \ldots, \mathscr{U}_{C_{5}}$. Легко видеть, что их объединение покрывает $Y$.
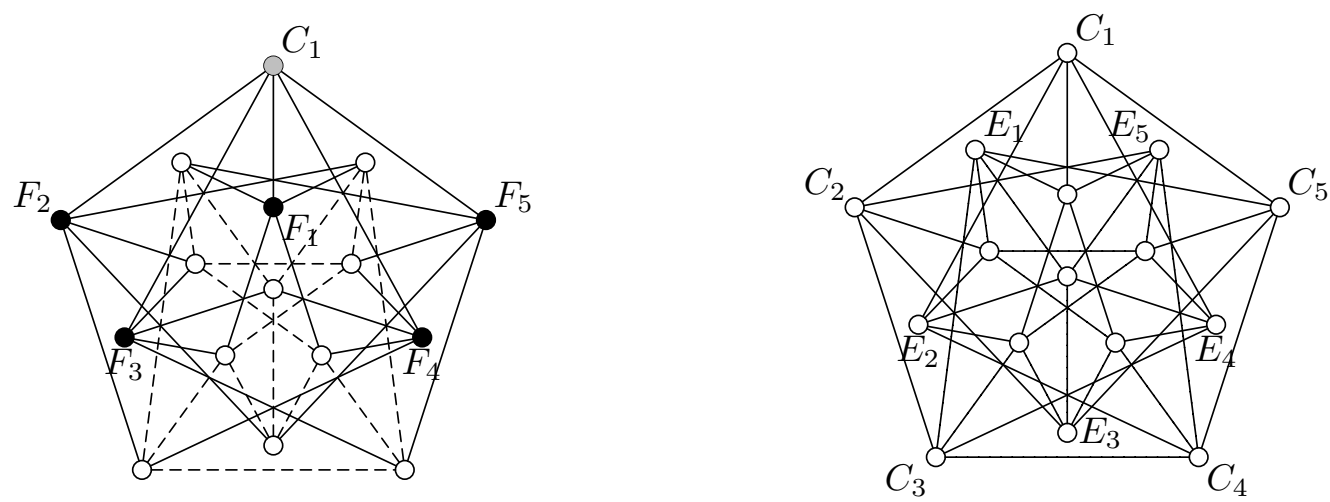

Рис. 3. Граф инцидентности (-1)-кривых на поверхности дель Пеццо степени 4. Слева отмечены прообраз квадрики $C_{1}$, задающий семейство цилиндров (серым), и стягиваемые $(-1)$-кривые (черным). Пунктирным ребрам отвечают пересечения $(-1)$-кривых, лежащие в цилиндрах семейства. Прочие четыре семейства цилиндров получаются симметричным образом при вращении графа и соответствуют $C_{2}, \ldots, C_{5}$. 
Пусть $W$ - собственное подмножество в $Y$, инвариантное относительно цилиндров всех семейств, а $w \in W-$ его произвольная точка. Можно считать, что $w$ лежит в цилиндре семейства $\mathscr{U}_{C_{1}}$. Тогда образ $\sigma_{1}(W) \subset \mathbb{P}^{2}$ инвариантен относительно семейства цилиндров $\left\{\sigma_{1}(U) \mid U \in \mathscr{U}_{C_{1}}\right\}$, каждый из которых является дополнением к квадрике $c$ и ее касательной. Известно, что через две точки на плоскости можно провести квадрику, касающуюся данной. Таким образом, для почти любой точки $x \in \mathbb{P}^{2} \backslash c$ существует слой некоторого цилиндра, проходящий через $x$ и $\sigma_{1}(w)$. А именно, точка $x$ не должна лежать на касательной к $c$, проходящей через $\sigma_{1}(w)$, и на квадриках, касающихся $c$ в точках раздутия и проходящих через $\sigma_{1}(w)$. Итак, $W$ плотно в $Y$. Аналогично $Y \backslash W$ плотно в $Y$, что невозможно. Значит, семейства $\mathscr{U}_{C_{1}}, \ldots, \mathscr{U}_{C_{5}}$ образуют трансверсальное покрытие поверхности $Y$.

4.2. Условие полярности. Обильные дивизоры $H$, для которых цилиндры из семейства $\mathscr{U}_{C_{1}}$ являются $H$-полярными, - это в точности обильные дивизоры из открытого конуса Ample $Y \cap\left\{\alpha_{1} F_{1}+\cdots+\alpha_{5} F_{5}+\alpha_{6} C_{1}+\alpha_{7} \sigma_{1}^{-1}(l) \mid\right.$ $\left.\alpha_{j}>0\right\}$ в $\operatorname{Pic} Y$. Определим такой конус для каждого $\mathscr{U}_{C_{i}}$ и обозначим его через Ample $\left(C_{i}, Y\right)$. Он не зависит от выбора касательной $l$ к $\sigma_{i}\left(C_{i}\right)$, поскольку по определению $l$ не проходит через центры раздутия. Тогда множество таких дивизоров $H$, что все цилиндры в $\bigcup_{i} \mathscr{U}_{C_{i}}$ являются $H$-полярными, будет открытым конусом $\bigcap_{i} \operatorname{Ample}\left(C_{i}, Y\right)$. Вычисления показывают, что он имеет 72 экстремальных луча, а именно:

$\begin{array}{lr}e_{0}, & 9 e_{0}-5 e_{i_{1}}-e_{i_{2}}-2 e_{i_{3}}-4 e_{i_{4}}-3 e_{i_{5}}, \\ 4 e_{0}-2 e_{i_{1}}-2 e_{i_{2}}-e_{i_{3}}-e_{i_{4}}-e_{i_{5}}, & 9 e_{0}-4 e_{i_{1}}-4 e_{i_{2}}-4 e_{i_{3}}-2 e_{i_{4}}-2 e_{i_{5}}, \\ 5 e_{0}-2 e_{i_{1}}-2 e_{i_{2}}-e_{i_{3}}-3 e_{i_{4}}-e_{i_{5}}, & 11 e_{0}-6 e_{i_{1}}-2 e_{i_{2}}-2 e_{i_{3}}-4 e_{i_{4}}-4 e_{i_{5}}, \\ 5 e_{0}-2 e_{i_{1}}-2 e_{i_{2}}-2 e_{i_{3}}-2 e_{i_{4}}, & 11 e_{0}-6 e_{i_{1}}-4 e_{i_{2}}-4 e_{i_{3}}-2 e_{i_{4}}-2 e_{i_{5}}, \\ e_{0}-2 e_{i_{1}}-2 e_{i_{2}}-2 e_{i_{3}}-2 e_{i_{4}}-2 e_{i_{5}}, & 11 e_{0}-6 e_{i_{1}}-2 e_{i_{2}}-4 e_{i_{3}}-4 e_{i_{4}}-4 e_{i_{5}}, \\ 6 e_{0}-2 e_{i_{1}}-2 e_{i_{2}}-3 e_{i_{3}}-e_{i_{4}}-3 e_{i_{5}}, & 11 e_{0}-6 e_{i_{1}}-4 e_{i_{2}}-4 e_{i_{3}}-4 e_{i_{4}}-2 e_{i_{5}}, \\ 7 e_{0}-4 e_{i_{1}}-2 e_{i_{2}}-2 e_{i_{3}}-2 e_{i_{4}}-2 e_{i_{5}}, & 15 e_{0}-8 e_{i_{1}}-2 e_{i_{2}}-4 e_{i_{3}}-6 e_{i_{4}}-6 e_{i_{5}}, \\ 9 e_{0}-5 e_{i_{1}}-3 e_{i_{2}}-4 e_{i_{3}}-2 e_{i_{4}}-1 e_{i_{5}}, & 15 e_{0}-8 e_{i_{1}}-6 e_{i_{2}}-6 e_{i_{3}}-4 e_{i_{4}}-2 e_{i_{5}},\end{array}$

где в качестве наборов $\left(i_{1}, \ldots, i_{5}\right)$ берутся циклические перестановки $(1,2,3,4,5)$.

Легко видеть, что антиканонический дивизор $\left(-K_{Y}\right)$ содержится в пересечении $\bigcap_{i} \operatorname{Ample}\left(C_{i}, Y\right)$. Аналогично теореме 6 получаем следующий результат.

Теорема 7. Пусть $Y$ - поверхность дель Пецио степени 4 и $\mathrm{H}$ - очень обильный дивизор, принадлежащий конусу $\bigcap_{i=1}^{5} \operatorname{Ample}\left(C_{i}, Y\right)$. Тогда аффинный конус AffCопе $_{H} Y$ является гибким. В частности, это верно для антиканонического дивизора $H=-K_{Y}$.

Мы выделили такой подконус в конусе обильных дивизоров, что содержащиеся в нем очень обильные дивизоры определяют гибкий аффинный конус. Однако он строго меньше обильного конуса. Вне этого подконуса лежит, например, класс обильных дивизоров $8 e_{0}-2 e_{1}-4 e_{2}-e_{3}-e_{4}-3 e_{5}$. Итак, вопрос о гибкости аффинного конуса над поляризацией поверхности дель Пеццо степени 4 по произвольному очень обильному дивизору остается открытым. 


\section{ЛитеРАТУРА}

[1] Ю. И. Манин, Кубические формы: алгебра, геометрия, арифметика, Наука, М., 1972.

[2] Р. Хартсхорн, Алгебраическая геометрия, Мир, М., 1981.

[3] I. Arzhantsev, H. Flenner, S. Kaliman, F. Kutzschebauch, M. Zaidenberg, Flexible varieties and automorphism groups, Duke Math. J., 162:4 (2013), 767-823.

[4] И. В. Аржанцев, М. Г. Зайденберг, К. Г. Куюмжиян, Многообразия флагов, торические многообразия и надстройки: три примера три примера бесконечной транзитивности, Матем. сб., 203:7 (2012), 3-30.

[5] I. Cheltsov, J. Park, J. Won, Affine cones over smooth cubic surfaces, http://arxiv. org/abs/1303. 2648.

[6] I. V. Dolgachev, Classical Algebraic Geometry: Modern View, Cambridge University Press, Cambridge, 2012.

[7] T. Kishimoto, Yu. Prokhorov, M. Zaidenberg, Group actions on affine cones, in: Affine Algebraic Geometry, CRM Proc. and Lecture Notes, vol. 54, Amer. Math. Soc., Providence, RI, 2011, 123-163.

[8] T. Kishimoto, Yu. Prokhorov, M. Zaidenberg, Unipotent group actions on Del Pezzo cones, J. Algebraic Geometry, в печати; http://arxiv.org/abs/1212.4479.

[9] R. Lazarsfeld, Positivity in Algebraic Geometry I. Classical setting: line bundles and linear series, Ergebnisse der Mathematik und ihrer Grenzgebiete, vol. 48, SpringerVerlag, 2004.

[10] W. A. Stein et al., Sage Mathematics Software (Version 4.6.1). The Sage Development Team, 2011; http://www.sagemath.org.

Кафедра высшей алгебры МГУ им. М. В. Ломоносова Институт Фурье, Гренобль, Франция

Поступило в редакцию e-mail: perepechko@mccme.ru 